\title{
Microencapsulation of gentamicin in biodegradable PLA and/or PLA/PEG copolymer
}

\author{
Y.-Y. HUANG $\dagger^{*}$ and T.-W. CHUNG \\ $\dagger$ Institute of Biomedical Engineering, College of Engineering, National Taiwan \\ University, Taipei, Taiwan \\ † Department of Biomedical Engineering, Chung-Yuan University, Chung-Li, \\ Taiwan
}

(Received 10 April 2000; revised 7 Fune 2000; accepted 9 August 2000)

\begin{abstract}
Biodegradable carriers containing gentamicin for local treatment of bone infection were developed. This paper describes the preparation and in vitro evaluation of these biodegradable implants. Poly-L-lactic acid (PLA) and polyL-lactic acid/polyethylene glycol (PLA/PEG) disk implants containing gentamicin sulphate were obtained by compression of microspheres prepared by a double emulsion process. The mean particle size distribution of the microspheres, based on volume, ranged from 95-270 $\mu \mathrm{m}$. The gentamicin sulphate loading of the microspheres, after a methylene chloride-water extraction procedure, exceeded $90 \%$ of the theoretical value. In vitro dissolution studies on the microspheres and implants with drug loadings $10-40 \% \mathrm{w} / \mathrm{w}$ indicated that the rate of drug release from both PLA and PLA/PEG implants increased, with an increase in drug loading. The release of gentamicin from microspheres was dependent on the properties of PLA and/or PLA/PEG. The PLA/PEG copolymer was more hydrophilic than the PLA homopolymer, and with a smaller $\mathrm{pH}$ change in the microenvironment with polymer being degraded. In comparison, the PLA/PEG implant released antibiotic faster and had a larger inhibitory zone based on the Bauer-Kirby experiments used to test the inhibitory activity of antimicrobial devices. Experimental results showed that the biodegradable PLA/PEG gentamicin delivery system had a potential for prophylaxis of post-operative infection.
\end{abstract}

Keywords: Microencapsulation, PLA, PEG Co-polymer, implant, gentamicin.

\section{Introduction}

Osteomyelitis is an inflammatory bone disease caused by microbial infection of the bone medullary cavity, cortex and/or periosteum. The bacteria reach the bone haematogenously or via direct inoculation. Direct inoculation occurs when bacteria is spread from a continuous focus of infection or by surgical or traumatic introduction of bacteria to the bone. One of the most common causes of osteomyelitis is post-operative sepsis following orthopaedic procedures. Prolonged systemic antibiotic therapy (parenteral or oral) for 4-6 weeks may be necessary for treatment (Waldvogel 1988).

Some disadvantages of prolonged parenteral therapy by intravenous or intramuscular antibiotic injections are the high cost of treatment, systemic toxicity and

* To whom correspondence should be addressed; e-mail: yyhuang@ha.mc.ntu.edu.tw 
patient discomfort. Oral antibiotic delivery may also be associated with patient compliance problems. In addition, because osteomyeolotis is often associated with bone destruction and limited vascularity at the infection sites, systemic therapy may fail to produce therapeutic tissue levels. Therefore, localized delivery of an antibiotic to the infected site was introduced to overcome the difficulties associated with systemic therapy. Local antibiotic therapy has the potential for reducing the morbidity associated with prolonged systemic antibiotic administration. The primary advantages of localized antibiotic delivery are that high local levels of antibiotic are achieved. It can avoid high dose administration, unlike oral or parenteral injection. Localized antibiotic delivery systems also reduce the possibility of building up drug resistance.

For local treatment, as well as prophylaxis of bone infections, nonbiodegradable polymethylmethacrylate (PMMA) bone cement or bead implants impregnated gentamicin have been used for decades (Wahlig et al. 1978, Klemm 1981). The release of gentamicin from PMMA beads is slow and incomplete (Wahlig et al. 1978, Sampath et al. 1992). As PMMA implants are not degradable, surgical removal of these implants is recommended (or necessary) when the drug is depleted. Furthermore, experimental data suggest that slime or glycocalyxproducing bacteria may adhere to the PMMA, even when this still contains antibiotic to which the bacteria are sensitive (Gristina and Costerton 1985). Pharmacokinetic studies of gentamicin-impregnated acrylic cement in 10 patients undergoing total hip joint arthroplasties indicated that only $5.78 \%$ of the total quantity implanted was released over 15 days (Bunetel et al. 1989). These disadvantages have generated interest in developing an absorbable carrying material for depot administration of antibiotics.

Biodegradable polymeric carriers, on the other hand, obviate the need for surgical removal of these carriers or implants at the end of therapy or when the drug is depleted. Polylactide and its copolymers have attracted much attention as materials for drug delivery (Lewis 1990). Jacob et al. (1991) reported the use of encapsulated microspheres of antibiotics in the prevention of infection in a rabbit open tibia fracture model. They found high local levels and low systemic levels of antibiotics, and low incidence of infection in rabbits treated with antibiotic encapsulated microspheres. Sampath et al. (1992) intended to fabricate poly(Llactic acid) (PLA) implants containing gentamicin sulphate for the treatment of osteomyelitis by prolonged localized delivery of drug. In their in vitro release test, cylind rical poly(L-lactic acid) implants obtained by compression of microcapsules, ranging from $178-444 \mu \mathrm{m}$, released an amount exceeding $80 \%$ gentamicin sulphate within 3 weeks. In contract, Miyamoto et al. (1993) pointed out that poly(DL-lactic acid) homopolymers with high molecular weights of 105000,21000 and 3300 were inappropriate as a bone morphogenetic protein (BMP) carrier, primarily because they produced strong foreign-body reactions or chronic inflammation, and were too slowly absorbed to be replaced by induced bone. In addition, poly(L-lactic acid) degrades in the body to lactic acid and creates a local acidic environment. The $\mathrm{pH}$ of a suspension of PLA (MW 650) in saline at a concentration of $1 \% \mathrm{wt} / \mathrm{wt}$ was 2.8. This event may exert an adverse effect on bone induction by BMP or bone formation, and must be considered in other applications whenever PLA homopolymers were applied.

Introducing polyethylene glycol into polylactic acid homopolymers could increase the degradation rate, decrease the acidity of degraded products, and 
increase the hydrophilicity of the polymer carriers. Hydrophilic PEG segments in the PLA copolymers may also enhance the diffusivity of water or drug in the polymer carrier (Zhu et al. 1990). Therefore, PLA/PEG copolymer may provide as a good matrix for depot administration of antibiotics.

In this study, biodegradable PLA, PLA/PEG carriers containing gentamicin for local treatment of bone infection were developed. The preparation, characteristics, and morphology of gentamicin-loaded microspheres, as well as the in-vitro release behaviour of disk implants made by these microspheres were studied. The prophylactic antimicrobial effect of the implant was also assessed.

\section{Materials and methods}

\section{Materials}

Gentamicin sulphate (Sigma Chemical Company, St Louis, MO, USA) was used as received. L-Lactide was purchased from DuPont Co. (Wilmington, DE, USA). PEG (molecular weight 2000) and PVA (degree of polymerization: 2000; viscosity of a $4 \%$ aqueous solution at $20^{\circ} \mathrm{C}$ : $35-45 \mathrm{cps}$ ) were obtained from Nacalai Tesque. Methylene chloride and chloroform, L.C. grade, were obtained from Alps Chemical Co. (Hsinchu, Taiwan). Polymer of poly-L-lactide (molecular weight 85 000-160 000) was also purchased from Sigma. All other regents were reagent HPLC grade, and used as received.

\section{Fabrication of PLA/PEG microspheres}

PLA/PEG copolymer was synthesized from L-lactide dimer and PEG 2000, under a nitrogen atmosphere, at $180^{\circ} \mathrm{C}$ (Huang et al. 1997). Gentamicin was encapsulated by a double emulsion, w/o/w emulsion technique, at room temperature. The aqueous drug solution $(1 \mathrm{ml})$ was first poured into the polymer solution (100 $\mathrm{mg}$ of polymer dissolved in $4 \mathrm{ml}$ methylene chloride or chloroform) to form a w/o emulsion. The w/o emulsion was then rapidly poured into $20 \mathrm{ml}$ of water containing $0.5 \mathrm{wt} \%$ of poly(vinyl alcohol). The mixture was sonicated for $15 \mathrm{~min}$ at $25^{\circ} \mathrm{C}$ to form an emulsion, then mechanically stirred. Stirring was continued until the organic solvent had evaporated. The microspheres were then separated, and dried under vacuum.

The particle size of microspheres was determined by a photon correlation spectroscope (He/Ne laser particle size analyser, Malvern Instrument, Worcestershire, UK). The ${ }^{1} \mathrm{H}-\mathrm{NMR}$ spectrum was measured with a Bruker AMX-400 MHz spectrometer using a solvent as the internal standard. The gentamicin sulphate loading of the microspheres was determined by a methylene chloride-water extraction procedure. Triplicate samples $(40 \mathrm{mg})$ of microspheres containing gentamicin sulphate were placed in methylene chloride to dissolve the PLA and PLA/PEG coat, and the drug was extracted five times with $6 \mathrm{ml}$ of distilled deionized water. Gentamicin sulphate in the aqueous phase was determined by TDx fluorescence polarization immunoassay (Abbot Laboratories). The final drug loading capacity of microspheres were $0.087 \mathrm{mg}$ gentamicin sulphate per $\mathrm{mg}$ of microspheres when the initial drug loading was $10 \%$. 


\section{Drug release test}

Microspheres, $0.1 \mathrm{~g}$, were poured into a $7 \mathrm{~mm}$ diameter die and compressed by $100 \mathrm{~kg} / \mathrm{cm}^{2}$ hydro-pressure forming a tablet, $7 \mathrm{~mm}$ in diameter and $3 \mathrm{~mm}$ in thick.

The release (dissolution) tests were carried out at $37^{\circ} \mathrm{C}$ in $0.025 \mathrm{M}$ phosphate buffer pH 7.4 brought to ionic strength 0.13 with sodium chloride under static conditions (one tablet in $5 \mathrm{ml}$ buffer). Drug concentration in the solution was assayed by fluorescence polarized immunoassay (TDx system).

\section{Morphology study}

The morphological difference of microspheres made of various PLA/PEG contents and resulting from different methods of encapsulation were monitored by scanning electron microscopy (Olympus JSM-35, Tokyo, Japan).

\section{In vitro antimicrobial activity test}

The antimicrobial activity of the getamicin drug delivery system was assayed by a Kirby-Bauer technique. $E$-coli was independently grown for $18 \mathrm{~h}$ in trypticase soy broth to a concentration of $0.5 \mathrm{McF}$ arland $\left(10^{8} \mathrm{cfu} \mathrm{m}^{-1}\right)$. A cotton swap placed in this suspension was rubbed across the surface of a Mueller Hinton agar plate. Tablets of individual drug delivery systems were pressed into the agar overlaid with the bacteria and incubated overnight at $37^{\circ} \mathrm{C}$. Zone sizes were assayed by measuring the distance to the centre of the tablet pressed into agar.

\section{Results and discussion}

Block copolymers of poly-L-lactic acid (PLA) and polyethylene glycol (PEG) were prepared by ring opening polymerization of L-lactide with polyethylene glycol, MW 2000, as previously described (Huang et al. 1997). The PEG weight per cent in the feed stream was 3 or $9 \%$, respectively. The PEG weight per cent in the resulting copolymer (i.e. $9 \%$ in the feed stream) was $14.1 \%$ as calculated from the ${ }^{1} \mathrm{H}-\mathrm{NMR}$ spectrum, as shown in figure 1 . The molecular weight (MW) of resulting copolymer was 11702 . The ratio of $\mathrm{PEG}$ in the resulting copolymers, PLA/PEG, was higher than the ratio in feed. This was probably due to the hydrophilicity of PEG in the reaction mixture that causes a reduction of reactivity of the lactide. Consequently, the copolymer molecular weights were lower than those of the homopolymers. As the PEG wt\% increased, the molecular weight and Tg decreased.

Double emulsion and emulsion solvent evaporation techniques were used in microencapsulating hydrophilic gentamicin sulphate. The particle size of gentamicin-loaded microspheres is shown in table 1.

The particle size of microspheres made from PLA homopolymer was larger than that made from PLA/PEG copolymer. PEG is an uncharged, linear and hydrophilic polymer. It has a good structural fit with $\mathrm{H}_{2} \mathrm{O}$, forming a gauche conformation suitable for hydrogen bonding between the water molecules and the ether oxygen of PEG in the ABA block copolymer. The high affinity of PEG with water pulled the PEG to the surface of micelles by the bulk water molecules in the emulsion process. At the same time, the hydrophobic 
PLA/PEG CDC13 112797

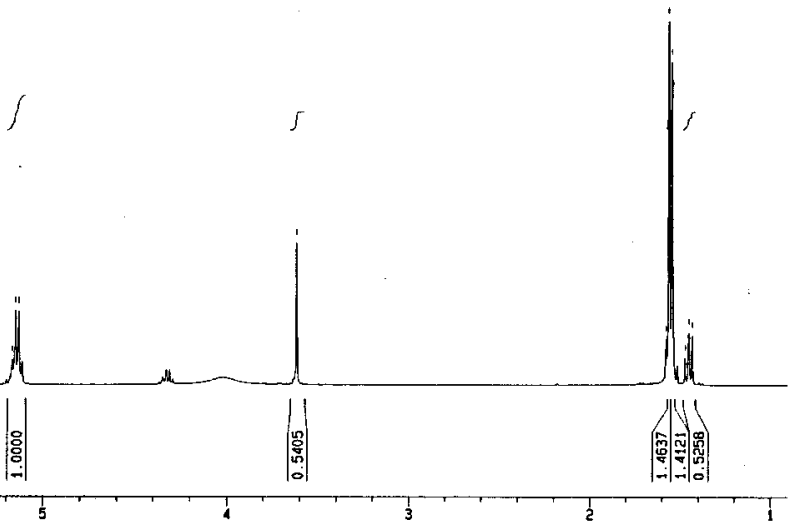

Figure 1. ${ }^{1} \mathrm{H}-\mathrm{NMR}$ spectra of the PLA/PEG (9\%) copolymer.

Table 1. The particle size of microspheres.

\begin{tabular}{lcc}
\hline Material & Drug loading & Particle size $(\mu \mathrm{m})$ \\
\hline PLA & $20 \%$ & 270.2 \\
PLA & $40 \%$ & 244.4 \\
PLA & $60 \%$ & 249.8 \\
PLA/PEG 3\%* & $20 \%$ & 165.6 \\
PLA/PEG 9\% & $20 \%$ & 133.9 \\
PLA/PEG 9\% & $40 \%$ & 95.21 \\
\hline
\end{tabular}

* PLA/PEG 3\% means that weight per cent in feed ratio of PLA:PEG during polymerization is 100:3.

polylactide segments were directed into the inner core of micelles. Core-corona type microspheres were formed after solidification. These core-corona type PLA/ PEG microspheres had hydrophilic steric brush structure. These particles showed less aggregation and were well dispersed. Under the light scattering method, particles would obtain smaller diameter. Another reason was that PEG was a surface-active agent too. Sometimes, it was used as an emulsifier in the literature. Due to the hydrophilicity of PEG segment in the copolymers or the release of unreacted PEG from the copolymer, the interfacial tension between the PLA/PEG polymer solution droplet and water in the emulsion will be lower than that of the PLA droplet in the emulsion, as shown in table 2. During the microsphereforming process, lower interfacial tension between the polymer solution and water will cause smaller droplets in the emulsion, then smaller microspheres were formed. 


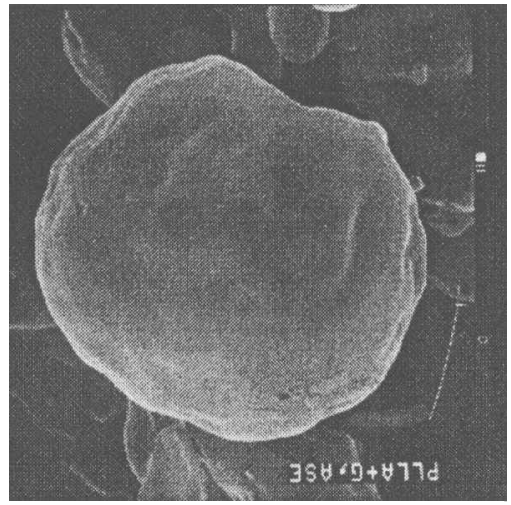

(a)

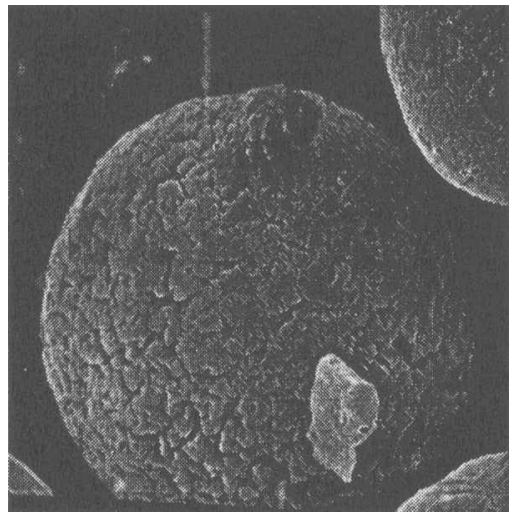

(b)

Figure 2. Microspheres containing gentamicin. (a) Microspheres made from PLA homopolymer, and $(b)$ microspheres made from PLA/PEG 9\% copolymer.

Table 2. The interfacial tension between the water/polymer in dichloromethane.

\begin{tabular}{llcc}
\hline Material & \multicolumn{3}{c}{ Interfacial tension $(\mathrm{mN} / \mathrm{m})$} \\
\hline Polymer concentration & $1 / 10$ & $1 / 50$ & $1 / 100$ \\
PLLA & 24 & 23.5 & 23.2 \\
PDLA & 27.3 & 26.3 & 25.1 \\
PLA/PEG 9\% & - & 16.9 & 20 \\
\hline
\end{tabular}

Figure 2 shows the morphology of microspheres made from PLA and PLA/ PEG copolymers. The surface of gentamicin-impregnated microspheres of PLA was smooth, but with ripple-like wrinkles. There were no visible pores on the surface, whereas many cracks were found on the surface of microspheres made from PLA/PEG. Each crack looks like an open channel. Owing to the higher hydration of PEG in the PLA/PEG copolymer, microspheres made from these copolymers were less well formed. High interactions between the PLA/PEG and the non-solvent (water in emulsion process) favoured the formation of macrovoids. The porosity of PLA/PEG microspheres is higher than that of PLA microspheres and is the total surface area of microspheres. In the partition-dependent diffusional transport polyester copolymer, a larger porous membrane increases the diffusion rate of the penetrant.

The mean particle size distribution of the microspheres, based on volume, ranged from $95-270 \mu \mathrm{m}$. The gentamicin sulphate loading of the microspheres, after a methylene chloride-water extraction procedure, exceeded $90 \%$ of the theoretical value. In vitro dissolution studies on the microspheres and implants with drug loading $10-40 \% \mathrm{w} / \mathrm{w}$ indicated that the rate of drug release from both PLA and PLA/PEG implants increased, while the dissolution half-life $\left(T_{50}\right)$ decreased exponentially with an increase in drug loading. Figure 3 shows the gentamicin release profiles from the PLA/PEG copolymer. PLA is hydrophobic, whilst PEG/PLA is more hydrophilic. Water easily interacts with the hydrophilic segments in the PEG/PLA copolymers and to hydrate the spheres. Drug release is 


\section{Gentamicin Release}
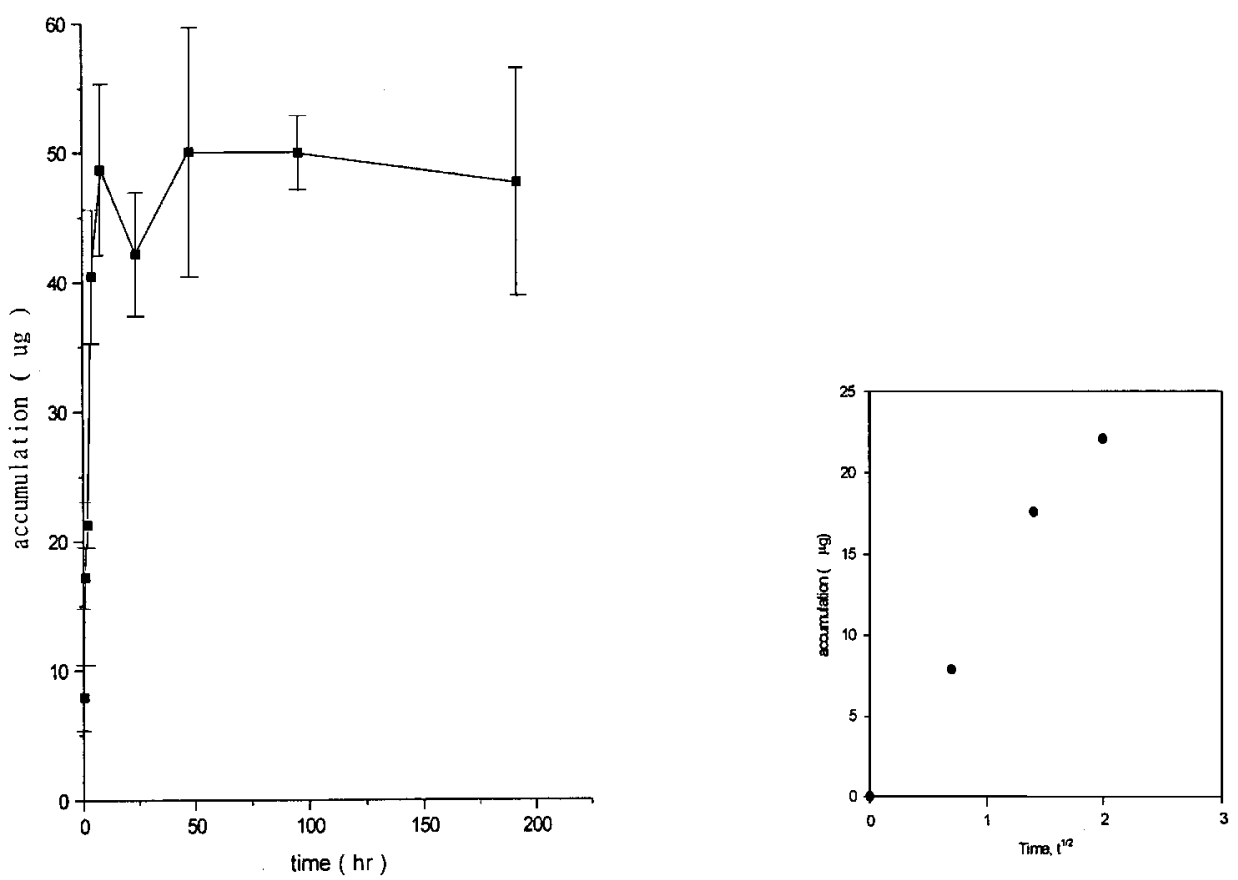

Figure 3. Drug release from PLA/PEG (3\% wt) copolymers. Large burst release shown in the initial period.

faster from the PEG/PLA copolymer than from the PLA homopolymer, because of the higher hydration rate and swelling characteristics of the ethylene glycol. Drug diffuses through the swollen matrix of the disc. If one re-plotted the data of release in the early stage versus $t^{1 / 2}$, as shown in the right-corner of figure 3 , the release curve showed a straight line that suggested the fraction of release was proportional to $t^{1 / 2}$. This means that gentamicin sulphate was principally releases by Fickian diffusion and that the erosion of the disc was negligible. For most biodegradable polyester polymers, diffusional transport is the dominating mechanism of release in the early stages. From the equation

$$
Q_{t}=2 C_{0}(D t / \pi)^{1 / 2}
$$

where $t$ is the release time; $D$ is the diffusivity; $C_{0}$ is the initial drug concentration in the disc, and $Q_{t}$ is the drug amount being released. The diffusivity of the disc could be calculated. A large burst was also observed in the dissolution test, as shown in figure 3 .

Figure 4 shows the in-vitro antimicrobial activity, the Kirby-Bauer technique, of the tablet fabricated from PLA microspheres $(a)$ and PLA/PEG microspheres (b), respectively. Tablets made of getamicine-impregnated microspheres were pressed into an agar plate overlaid with E. coli and incubated overnight at $37^{\circ} \mathrm{C}$. The tablets had zones of inhibition with diameters of $25.6 \mathrm{~mm}$ (PLA) and $30.8 \mathrm{~mm}$ 


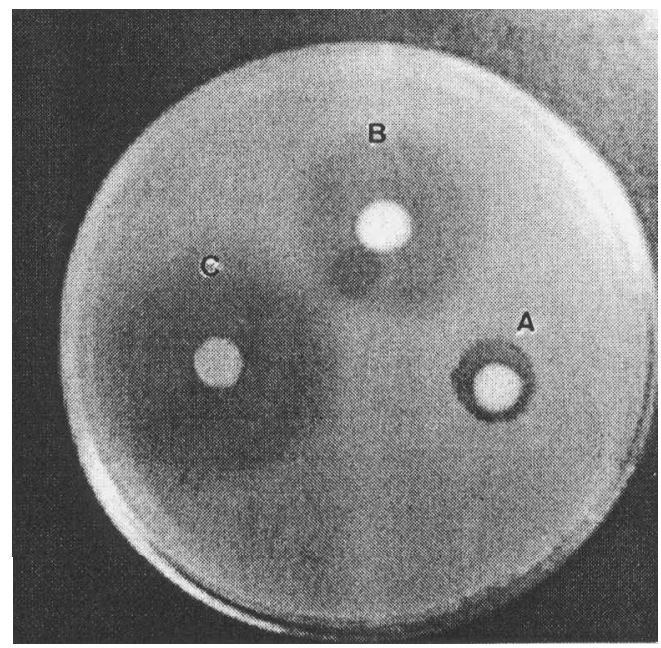

Figure 4. The Kirby-Bauer technique showing a tablet made of getamicine-impregnated microspheres pressed into an agar plate overlaid with E. Coli and incubated overnight at $37^{\circ} \mathrm{C}$. (a) control: PLA/PEG 3\% only; (b) PLA microsphere containing gentamicin; (c) PLA/PEG 3\% microspheres containing gentamicin.

(PLA/PEG 3\%). The inhibition zone of a PLA/PEG tablet was bigger than the inhibition zone of a PLA tablet, because the release rate of drug from the PLA/ PEG microspheres was faster. The control tablet also showed a slight bactericidal effect, probably due to the result of residual methylene chloride. It is necessary to remove methylene chloride from the dosage form completely. The presence of this organic solvent even in a small quantity may cause irritation in that target tissue, which is already inflamed.

\section{Conclusions}

Biodegradable PLA/PEG copolymers were polymerized by ring polymerization. The PLA/PEG copolymer was more hydrophilic than the PLA homopolymer, and had a lower glass transition temperature. The microspheres made from PLA/PEG were smaller than those from PLA. The surface of microspheres made from PLA/PEG was not as smooth as that of PLA. Gentamicin release from microspheres was effected by the properties of PLA and/or PLA/PEG copolymers. The in-vitro drug release tests and Bauer-Kirby tests showed that the biodegradable PLA/PEG gentamicin delivery system has potential in the applications for the treatment and prophylaxis of postoperative infection.

\section{Acknowledgements}

This research was supported by the National Science Council and Chinese Petroleum Cooperation, Taipei, Taiwan NSC 88-CPC-E-002-026. 


\section{References}

Bunetel, L., Segui, A., Cormier, M., Percheron, E., and Langlais, F., 1989, Release of gentamicin from acrylic bone cement. Clinical Pharmacokinetics, 17, 291-297.

Gristina, A. G., and Costerton, J. W., 1985, Bacterial adherence to biomaterials and tissue. The significance of its role in clinical sepsis. Fournal of Bone and Foint Surgery, 67, 264-273.

Huang, Y. Y., Chung, T. W., and Tzeng, T. W., 1997, Drug release from PLA/PEG microparticulates. International Fournal of Pharmaceutics, 156, 9-15.

Jасов, E., Setterstrom, J. A., Васh, D. E. et al., 1991, Evaluation of biodegradable ampicillin amhydrate microcapsules for local treatment of experimental staphylococcal osteomyelitis. Clinical, Orthopaedic and Related Research, 267, $237-244$.

KLemM, K., 1981, Septopal-a new way of local antibiotic therapy. In Local antibiotic treatment in osteomyelitis and soft-tissue infections, edited by T. J. G. Van Rens and F. H. Kayser (Amsterdam: Excerpta medica), pp. 24-31.

LewIS, D. H., 1990, Controlled release of bioactive agents from lactide/glycolide polymers. In Biodegradable Polymers as Drug Delivery Systems, edited by N. Chasin and R. Langer (New York: Dekker), pp. 1-41.

Miyamoto, S., Takaoka, K., Okada, T., Yoshikawa, H., Hashimoto, J., Suzuki, S., and Ono, K., 1993, Polylactic acid-polyethylene glycerol block copolymer-A new biodegradable synthetic carrier for bone morphogenetic protein. Clinical, Orthopaedic and Related Research, 294, 333-343.

Sampath, S. S., Garvin, K., and Robinson, D. H., 1992, Preparation and characterization of biodegradable poly(L-lactic acid) gentamicin delivery systems. International Fournal of Pharmaceutics, 78, 165-174.

Wahlig, H., Dingeldein, E., Bergmann, R., and Reuss, K. 1978, The release of gentamicin from polymethylmethacrylate beads. An experimental and pharmacokinetics study. Fournal of Bone and Foint Surgery, 60, 270-275.

Waldvogel, F. A., 1988, Acute osteomyelitis. In Orthopaedic Infection, edited by D. Schlossberg (New York: Springer), pp. 1-20.

Z HU, K. J., Lin, X., and YANG, S., 1990, Preparation, characterization, and properties of polylactide(PLA)-poly(ethyleneGlycol)(PEG) copolymers: a potential drug carrier. Fournal of Applied Polymer Science, 39, 1-9. 